\section{Cureus}

Review began 09/17/2012 Published 12/11/2012

\section{(c) Copyright 2012}

Engin et al. This is an open access article distributed under the terms of the Creative Commons Attribution License CC-BY 3.0., which permits unrestricted use, distribution, and reproduction in any medium, provided the original author and source are credited.

\title{
Robotic Radiosurgery for Liver Metastases
}

\author{
Kayihan Engin ${ }^{1}$, Nadir Kucuk ${ }^{2}$, Murat Dokdok ${ }^{3}$, Tayfun Enunlu ${ }^{4}$, Kezban Berberoglu ${ }^{5}$, \\ Hande Bas. Ayata ${ }^{6}$, Cemile Ceylan ${ }^{7}$, Ayhan Kilıc ${ }^{6}$, Metin Guden ${ }^{6}$ \\ 1. Radiation Oncology Department, Anadolu Medical Center, Kocaeli, Turkey, Radiation Oncology \\ Department, Anadolu Medical Center, Kocaeli, Turkey 2. Radiation Oncology Department, Anadolu \\ Medical Center 3. Radiology Department, Anadolu Medical Center, Kocaeli, Turkey, Radiology \\ Department, Anadolu Medical Center, Kocaeli, Turkey 4. Statistics Department, Anadolu Medical Center, \\ Kocaeli, Turkey, Statistics Department, Anadolu Medical Center, Kocaeli, Turkey 5. Nuclear Medicine, \\ Anadolu Medical Center, Kocaeli, Turkey, Nuclear Medicine, Anadolu Medical Center, Kocaeli, Turkey 6. \\ Radiation Oncology, Anadolu Medical Center, Kocaeli, Turkey, Radiation Oncology, Anadolu Medical \\ Center, Kocaeli, Turkey 7. Radiation Oncology, Istanbul Oncology Hospital
}

$\square$ Corresponding author: Nadir Kucuk, nadirk70@hotmail.com

Disclosures can be found in Additional Information at the end of the article

\section{Abstract}

Purpose: The prevalence of liver metastases is very high for patients whose primary diagnosis are colorectal, pancreas, lung, and breast cancer. Local control of the disease may increase both quality of life and survival. The aim of this study to show feasibility, efficiency, and low morbidity combined with a high local control probability in use of robotic stereotactic body radiation therapy (rSBRT) treatment for liver metastases.

Methods and Materials: A total of 55 patients with 84 liver metastases from different primary sites who underwent rSBRT in our center between July 2006 and December 2011 were reviewed for this study. Patients who refused surgery or judged inoperable were included in this study. Patients were required to have adequate liver function and more than three months minimum life expectancy. An average of three (3-5) fiducial markers were implanted percutaneously under CT-guidance with local anaesthesia. Maximum three liver metastases from any primary sites were treated and accepted for this study. Treatment planning CT, magnetic resonance imaging (MRI), and positron emission tomography-CT (PET-CT) were used for tumor delination. Treatment plan dose was prescribed at the PTV with an isodose line range 70-85\% which was covering the $95 \%$ volume of PTV. We mandated that minumum $700 \mathrm{cc}$ of normal liver had to receive at total dose less than $15 \mathrm{~Gy}$ in three to five fractions. The patients were examined every three months after rSBRT by biochemical examinations and imaging. PET-CT or MRI were performed at each follow-up.

Results: Fifty-five patients (32 males, 23 females) who had liver metastases from various primary sites were treated with rSBRT using real-time tumor tracking system. Mean age of patients was 61 years (range 22 to 86 years). The primary sites included colorectal $(n=23)$, pancreas $(n=8)$, breast $(n=7)$, lung $(n=6)$, bladder $(n=3)$, and others $(n=8)$. The total prescribed dose was $45 \mathrm{~Gy}$ (range $24 \mathrm{~Gy}$ to $45 \mathrm{~Gy}$ ) in median 3 (range three to six) fractions. The minimum normal liver volume (700 cc) was received mean $7.8 \mathrm{~Gy}$ (range $2 \mathrm{~Gy}$ to $14.6 \mathrm{~Gy}$ ). Forty-nine (89\%) patients were evaluated for tumor response, local control and survival analysis. The median follow-up was 12 months (range four to 39 months). CR was seen in 25 (51\%) patients, PR in 12 (25\%) patients, and SD in two (4\%) patients at first follow-up. Ten (10\%) patients had progressed inside the treated volume at first follow-up. The actuarial local control rate for patients is shown in Figure 2. The actuarial one-year and two-year local control rates were 61\% (95\% confidence interval (CI), 53\%-69\%) and 39\% (95\% CI, 28\%-49\%), respectively. The median overall survival was 16 months. The actuarial one-year and two-year overall survival rates were 
Conclusion: The results of our study have show that rSBRT for the treatment of liver metastases is feasible and safe with minimal side-effects. Further studies are required with larger patient series to compare rSBRT with other local ablative techniques and systemic therapies.

Categories: Internal Medicine, Radiation Oncology, Radiology

Keywords: stereotactic radiosurgery, body radiation, srs, liver metastases, cyberknife $\subset$

\section{Introduction}

The liver is one of largest organs in the body which is made of hepatocytes, located right-hand portion of the abdominal cavity, divided into two lobes. Main functions of the liver are to produce bile, which helps carry away waste and break down fats in the small intestine during digestion and to produce certain proteins for blood plasma.

Approximately eighty thousand new liver tumors are diagnosed per year in the United States, and both primary or metastatic tumors of the liver generally respond poorly to all types of treatment modalities [1-3]. The decision of the optimal treatment modality in patients with liver metastases depends on the general clinical data, such as primary tumor type, imaging characteristics of the liver, number of the lesions, size, and location of the lesions, etc. A mapping of metastases in the liver is also very important to define volume of the uninvolved liver segments. The size of the uninvolved liver volume is very critical before decision of treatment modalities.

The prevalence of the liver metastases is very high for patients whose primary diagnoses are colorectal, pancreas, lung, and breast carcinomas. Local control of the disease may increase both quality of life and survival. Surgical resection of the liver metastases is known to be a first therapeutic option in patients with solitary and limited number of metastases [4-5]. However, resection cannot be carried out in the majority of these metastases, and only 10-25\% of those patients are candidates for curative resection [5-7]. Morbidity and mortality rates after hepatic resection were reported as high as $35 \%$ and $3 \%$ [8-9]. Chemotherapy is also efficient method to treat the liver lesions which cannot be surgically removed. New chemotherapeutic agents and targeted therapies have been commonly used over the past decade and significant improvements in results for some primary sites have been observed [10-12]. Unfortunately, the results of systemic therapies need improvement for the majority of primary sites and liver metastases still remain as a difficult therapeutic challenge [4]. Several local treatment modalities, including cryosurgery, radiofrequency ablation (RFA), external/internal beam irradiation, and laser-induced thermal therapy, have been used to treat these metastases. RFA is the most common method used in practice for treatment of liver metastases. This method has some limitations, such as tumor size (smaller than $3 \mathrm{~cm}$ ) and proximity of major biliary/vascular structures [13-14].

Treatment of intrahepatic metastases by radiotherapy had a limited role in the past because of the low tolerance of the whole liver to irradiation. New radiotherapy techniques, such as stereotactic body radiation therapy (SBRT), allows delivering high dose of radiation in a single session or limited number of sessions to a confined area, while sparing surrounding critical organs and other healthy tissues. With the development of robotic stereotactic body radiation therapy (rSBRT) device, it is possible to track tumor motion during in the treatment by using implanted fiducial markers combined with respiratory motion modeling. However, only a few number of studies have been published to evaluate the efficiency of rSBRT for liver metastases $[7,15-17]$. The aim of this study to show feasibility, efficiency and low morbidity combined with 


\section{Cureus}

a high local control probability in use of rSBRT treatment for liver metastases.

\section{Materials And Methods}

\section{Patient selection}

A total of 55 patients with 84 liver metastases from different primary sites who underwent rSBRT in our center between July 2006 and December 2011 were reviewed for this study. Primary site of malignancy for patients are listed in Table 1.

\begin{tabular}{|l|l|l|}
\hline Primary origin & $\mathbf{n}$ & $\%$ of patients \\
\hline Breast & 7 & $13 \%$ \\
\hline Pancreas & 8 & $14 \%$ \\
Colorectal & 23 & $41 \%$ \\
Lung & 6 & $11 \%$ \\
Bladder & 3 & $5 \%$ \\
Stomach & 2 & $4 \%$ \\
Ovary & 1 & $2 \%$ \\
\hline Cervix & 1 & $2 \%$ \\
\hline Head and neck & 2 & $4 \%$ \\
Liver (Cholangiocarcinoma) & 1 & $2 \%$ \\
\hline Sarcoma & 1 & $2 \%$ \\
\hline
\end{tabular}

\section{TABLE 1: Primary origins of tumors treated with rSBRT}

Treatment decision of rSBRT was determined by evaluation of a surgeon, a radiologist, a radiation oncologist, and medical oncologist. Patients who refused surgery or had been judged inoperable were included in this study. Patients were required to have adequate liver function and more than three months minimum life expectancy. Other inclusion criterias were maximum tumor diameter $<6 \mathrm{~cm}$, Karnofsky performance status $>60$, no active liver infection, and no prior radiotherapy to the liver. Maximum three liver metastases from any primary sites were treated and accepted for this study. Patient and tumor characteristics are summarized in Table 2.

\section{Characteristic}

\# of Patients

\# of treated lesions

\section{Value}

55

84

37 pts $(67 \%)$ 


\section{Cureus}

2 lesions

3 lesions

7 pts $(13 \%)$

11 pts $(20 \%)$

Gender

Male

32 pts $(58 \%)$

Female

23 pts $(42 \%)$

Age

Median

Mean

Range

Time from primary diagnosis to first metastasis

Median

Range

Time from first liver metastasis to rSBRT

Median

Range

Metastases except liver during rSBRT

None

Multiple ( $\geq 2$ different sites)

Lung

Bone

Other sites (single location)

Received chemotherapy during rSBRT

Yes

No

Mean

Range

Tumor volume
59 years

60.7 years

22-86 years

17 months

0-151 months

6 months

0-15 months

33 pts $(60 \%)$

4 pts $(7 \%)$

9 pts $(17 \%)$

5 pts $(9 \%)$

4 pts $(7 \%)$

44 pts $(80 \%)$

11 pts $(20 \%)$
$40,48 \mathrm{cc}$

$3.8 \mathrm{cc}-336 \mathrm{cc}$

TABLE 2: Patient and tumor characteristics 


\section{Pre-treatment preparations}

An average of three (three to five) fiducial markers (Civco Medical Solutions, Kalona, Iowa) were implanted percutaneously under CT-guidance with local anaesthesia. Fiducials were made of gold and cylindrical-shaped. These were implanted in or near tumor volume by keeping minimum distance between the fiducials of $2 \mathrm{~cm}$. After implementation, all patients were kept waiting minimum a week to check the possibility of fiducial migration. Patients were immobilized in supine position by using whole body vacuum cradle immobilization device and fitted with the Synchrony vest. Computed tomography (CT) images were recorded with same immobilized position and vest without using contrast material. CT images were taken in $1 \mathrm{~mm}$ slice thickness using breath-holding technique. In addition, T1-weighted magnetic resonance images were scanned using contrast material for all patients.

\section{Treatment planning and delivery}

Treatment planning CT, magnetic resonance imaging (MRI), and positron emission tomography-CT (PET-CT) were used for tumor delination. All these scans were imported into the treatment planning system (Multiplan, version 2.2.0) and fused digitally, often with CT images, PET-CT scans, or MRI images. Contouring was done using only CT/MRI fusion in six (11\%) patients. Treatment planning CT was alone used for contouring in one patient. CT/PET$\mathrm{CT}$ and CT/MRI fusions were used for accurate identification of the location of the gross tumor volume (GTV) in other 48 (87\%) patients. The planning target volume (PTV) was defined as a 5 $\mathrm{mm}$ uniform margin around GTV to account for possible microscopic disease and targeting uncertanity. The liver, kidneys, spinal cord, lungs, stomach, duodenum, and intestine were contoured in planning. Published reports have showed that minimum volume of $700 \mathrm{cc}$ of liver should remain uninjured by SBRT (18-19). We mandated that minimum $700 \mathrm{cc}$ of normal liver had to receive at total dose less than $15 \mathrm{~Gy}$ in three to five fractions. Dose constraints for organs at risk (OARs) were applied in all treatment plans as listed in Table 3. Treatment plan dose was prescribed at the PTV with an isodose line range $70-85 \%$ which was covering the $95 \%$ volume of PTV. CyberKnife (Accuray, Inc., Sunnyvale, CA) was used for rSBRT. Tumor tracking was performed using the Synchrony system (Accuray) during rSBRT and the total treatment time was approximately 50-180 min, with most patient treated within $100 \mathrm{~min}$. 


\section{Cureus}

\section{Critical Structure}

Liver

Kidneys

Spinal cord

Stomach

Duedenum

Bowel

Lungs

Heart

\section{Dose Constraint}

$\geq 700$ cc $\leq 15$ Gy

V5<\%75, Dmax $<15$ Gy

Dmax $<18$ Gy

V20<\%5, Dmax $<25$ Gy

V20<\%5, Dmax $<25$ Gy

V20<\%5, Dmax <25 Gy

V15 $<5$, Dmax $<18$ Gy

Dmax $<25$ Gy

Vn-Organ volume that receives a dose of $\mathrm{n}$ Gy or less,

Dmax- Maximum point dose in the organ

\section{TABLE 3: Dose constraints for OARs}

\section{Follow-up and toxicity}

The patients were examined at three month intervals after rSBRT by biochemical examinations and imaging. PET-CT or MRI were performed at each follow-up. Response was evaluated according to RECIST (Response Evaluation and Criteria in Solid Tumors) criteria [20]. Complete response (CR) was defined as the complete disapperance of the target lesion. Partial response (PR) was defined as at least a 30\% decrease in the longest diameter of the target lesion. Progressive disease (PD) was defined as at least $20 \%$ increase in the longest diameter of the target lesion. Stable disease (SD) was defined as a tumor status that no any changes in tumor dimensions or vascular modification. Local control was defined as the absence of tumor progression within or at the border of the radiation field based on imaging. Local recurrence was defined as failure within treated volume or at the border of the radiation field. Toxicity was also scored using National Cancer Institute (NCI) Common Terminology Criteria for Adverse Events (CTCAE) v3.0 [21]. Toxicity which was occurred in the first three months after rSBRT were termed as acute toxicity. Events which occurred after three months from rSBRT were termed as late toxicity.

\section{Statistical analyses}

SPSS 17 (SPSS Inc, Chicago, IL) software was used for the statistical analyses. Fisher's exact test and Log Rank were used to assess relation between category variables. The rates of actuarial overall survival, actuarial local control, actuarial survival by primary tumor types, and actuarial survival by number of treated lesion(s) were calculated by the Kaplan-Meier survival curve method. All parameters were tested by univariable and multivariable regression model. Cox regression model was used to express the factor effecting local control and overall survival.

\section{Results}




\section{Cureus}

\section{Patient population}

Fifty-five patients (32 males - 23 females) who had liver metastases from various primary sites were treated with rSBRT using real time tumor tracking system. The mean age of patients was 61 years (range 22 to 86 years). Primary sites included colorectal $(n=23)$, pancreas $(n=8)$, breast $(n=7)$, lung $(n=6)$, bladder $(n=3)$, and others $(n=8)$. Of the three patients who had two liver metastases in the same lobe, single treatment plan was created and delivered. Of the two patients who had three liver metastases in the same lobe, single treatment plan was created and delivered. Eighty-four lesions derived from different primary sites in 55 patients were treated with totally 77 treatment plans. Median time from primary tumor diagnosis to first liver metastases was 17 months (ranging between 0 and 151 months). Median time from first liver metastases to rSBRT was six months (range of 0-15 months). Of the 33 (60\%) patients treated with rSBRT, there was no other organ metastases, except liver. Nine (17\%) patients had only lung and five (9\%) patients had only bone metastases, except liver lesions during in rSBRT. Four (7\%) patients have only one metastases, except lung or bone. Four (7\%) patients had multiple ( $\geqslant 2$ different organ) metastases except liver lesions during treatment. One-fifth of patients, 11 (20\%), didn't receive any concurrent systemic therapy during irradiation (Table 2).

\section{Dose-volume characterictics}

The dose-volume characteristics of treatments are listed in Table 4. Median collimator size was $20 \mathrm{~mm}$ (range: $10 \mathrm{~mm}$ to $35 \mathrm{~mm}$ ). The total prescribed dose was $45 \mathrm{~Gy}$ (range: $24 \mathrm{~Gy}$ to $45 \mathrm{~Gy}$ ) in median three (range: three to six) fractions. The radiation dose was prescribed to median 80\% (range: $70 \%$ to $85 \%$ ) isodose line. The median percent of the PTV covered by the prescription isodose line was $96.6 \%$ (range: $90 \%$ to $99 \%$ ). Mean number of beam used for rSBRT was 224 (range: 117 to 314). The minimum normal liver volume (700 cc) received mean $7.8 \mathrm{~Gy}$

(range: 2 Gy to $14.6 \mathrm{~Gy}$ ).

\section{Characteristic}

Value

Total liver volume

Mean

$1571,8 \mathrm{cc}$

Collimator size

Median

$20 \mathrm{~mm}$

Range

$10 \mathrm{~mm}-35 \mathrm{~mm}$

Prescription dose

Median

Range

\# of fractions

Median

Range

\section{3-6}

Prescibed isodose line
Median

Range
45 Gy

24 Gy-45 Gy 


\section{Cureus}

Conformity index

$\begin{array}{ll}\text { Median } & 1.22\end{array}$

Range $\quad 1.06-1.94$

Homogeneity index

$\begin{array}{ll}\text { Median } & 1.25 \\ \text { Range } & 1.18-1.43\end{array}$

Tumor coverage

Median $\quad 96.6 \%$

Range $\quad 89.8 \%-99.5 \%$

\# of beams

$\begin{array}{ll}\text { Mean } & 224\end{array}$

Range 117-314

700 cc normal liver tissue dose

Mean $\quad 7.8$ Gy

Range 2 Gy-14.6 Gy

TABLE 4: Dose-volume characteristics of rSBRT

\section{Clinical outcome}

Fifty-five patients were accepted and evaluated for this study. Six patients had no follow-up images in our system. Tumor response rates, local control, and survival analysis for 49 (89\%) patients are listed in Table 5 . 


\section{Cureus}

\section{Variable}

Patients (n)

Alive (n)

$\operatorname{Dead}(\mathrm{n})$

Follow up (mo)

Median

Range

Response in first follow up

CR
PR
SD
PD

$25(51 \%)$

Median

10

Range

6-23

Overall survival (mo)

\section{Value}

49

22

27

$12(25 \%)$

$2(4 \%)$

$10(20 \%)$

Local recurrence interval (mo)

Median

\section{TABLE 5: Clinical evaluation of patients after rSBRT}

Twenty-seven (55\%) patients died, and 22 (45\%) were alive at the time of our evaluation. The median follow-up was 12 months (range: four to 39 months). CR was seen in 25 (51\%) patients, PR in 12 (25\%) patients and SD in two (4\%) patients at first follow-up. Ten (20\%) patients progressed inside the treated volume at first follow-up. PD was seen in 17 (35.4\%) patients at second follow-up. Percentage of tumor response (CR, PR or SD) rate was decreased from $80 \%$ to $63 \%$ at the time of second follow-up. An example of CR with continued local control at second follow-up is shown in Figure 1.
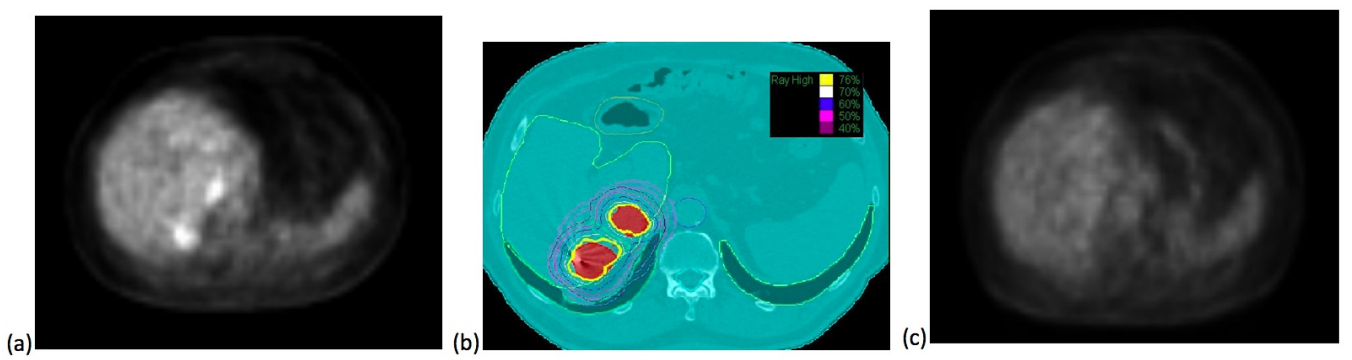


\section{Cureus}

\section{FIGURE 1: An example of CR with continued local control in a patient with metastatic colorectal cancer}

An example of $\mathrm{CR}$ with continued local control in a patient with metastatic colorectal cancer (a) axial view of lesions in PET before rSBRT, (b) treatment planning showing dose distrubition, (c) PET image after six months from rSBRT

Logistic regression model was used for evaluating effect of different parameters to tumor response. Age, gender, location of primary tumor, tumor size, number of fraction, total dose, receiving concurrent chemotherapy and rSBRT, presence of other metastases, except liver, and number of treated lesions were analyzed using logistic regression model. Location of primary tumor is the only parameter that affects tumor response. Colorectal tumor metastases had better results than other tumor metastases in the first follow-up ( $\mathrm{p}=0.05)$. The effect of other organ metastases to tumor response was evaluated. Of the 19 (39\%) patients who had other organ metastases, except liver, progression was seen in two patients. Of the 30 (61\%) patients who had no other organ metastases, except liver, progression was seen in eight patients. No correlation was found statistically ( $\mathrm{p}=0.16$, Fisher's Exact Test) between tumor response and presence of other organ metastases, except liver. The effect of receiving chemotherapy during rSBRT to tumor response was observed. No progression was seen in all eight (16\%) patients who had no systemic therapy during rSBRT. Of the 41 (84\%) patients who had received chemotherapy during rSBRT, progression was seen in 10 patients. There was no significant correlation statistically ( $\mathrm{p}=0.14$, Fisher's Exact Test) between tumor response and receiving chemotherapy during rSBRT.

The actuarial local control rate for patients is shown in Figure 2.

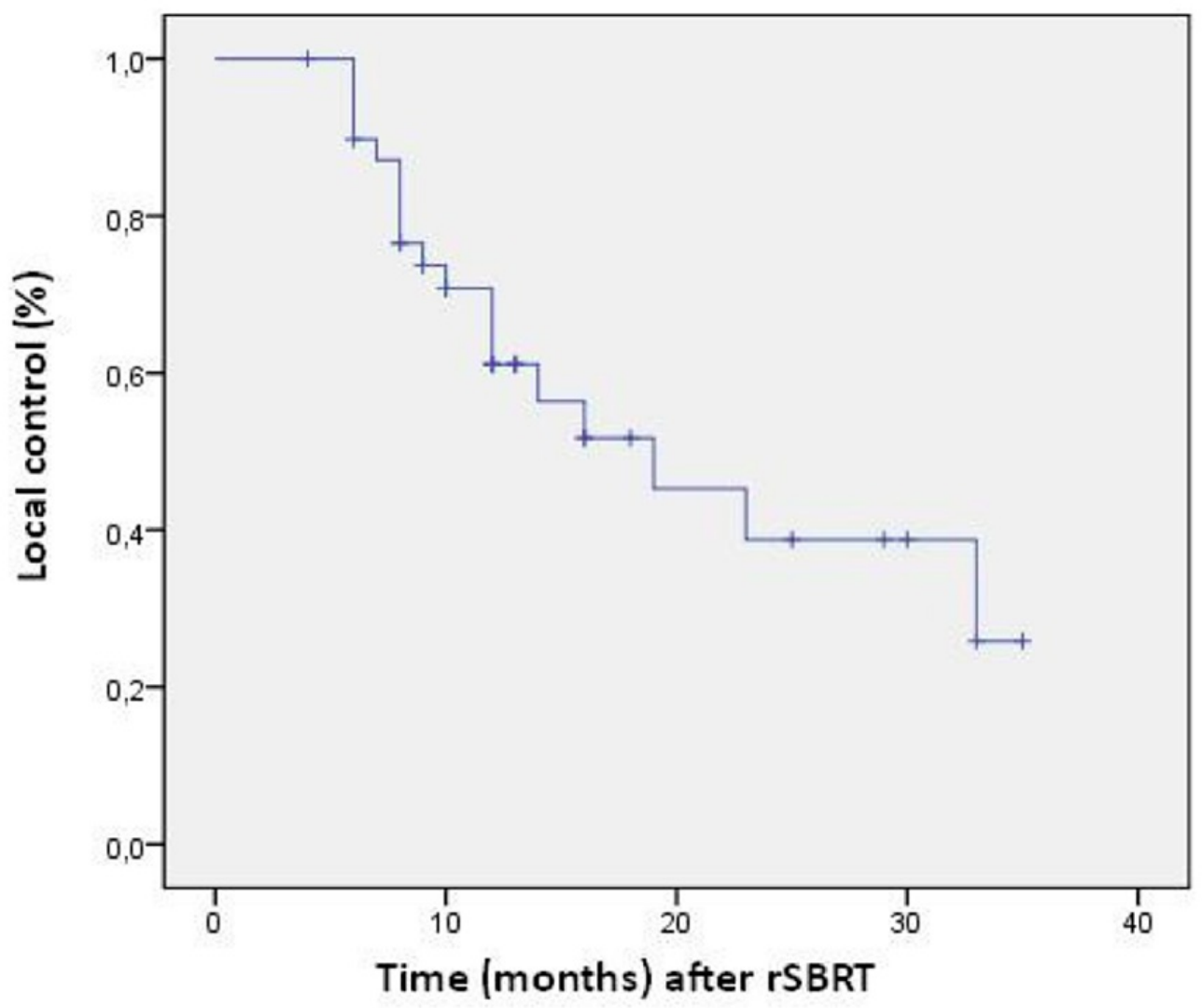




\section{Cureus}

\section{FIGURE 2: Actuarial local control rate for patients evaluated by}

the Kaplan- Meier method.

The actuarial one-year and two-year local control rates were $61 \%$ (95\% confidence interval (CI), 53\%-69\%) and 39\% (95\% CI, 28\%-49\%), respectively. Of the complete responding patients at the first follow-up, developed local recurrence in 12 (48\%) of them, with a median time to relapse of 10 months (range six months to 23 months). In two patients who had PR at first follow-up, CR was seen at second follow-up. Kaplan Meier univariate analysis and Cox regression multivariate analysis were used for evaluating local control. Results are seen in Table 6 .

Variable

Age

$$
\leq 60
$$

$>60$

Gender

$$
\text { Male }
$$

0.073

2.535

Female

\# of treated lesion(s)

$$
1 \text { or } 2
$$

0.005

8.543

\section{3}

Tumor size

$$
\leq 30 \mathrm{~mm}
$$

$$
>30 \mathrm{~mm}
$$

\# of fraction

$$
\leq 3
$$$$
>3
$$

Total dose

$$
\leq 30 \text { Gy }
$$

0.84

0.910

1.081

$>30$ Gy

Primary histology

Colorectal

0.548

Noncolorectal

0.053

0.018

8.084

\section{.226}

535

Odds ratios 


\section{Cureus}
Yes
0.105
0.76
0.857
No

Concurrent chemoterapy

Yes

0.407

0.853

0.875

No

TABLE 6: Kaplan Meier univariate and Cox regression multivariate analysis for local control

Age, number of treated lesion(s) and number of fraction was statistically important for local control according to Cox regression multivariate analysis. Patients with $>60$ years old and one or two metastases and patients who received $\leqslant 3$ fractions had better local control according to our statical results.

The median overall survival was 16 months. The actuarial overall survival rates for patients are shown Figure 3.

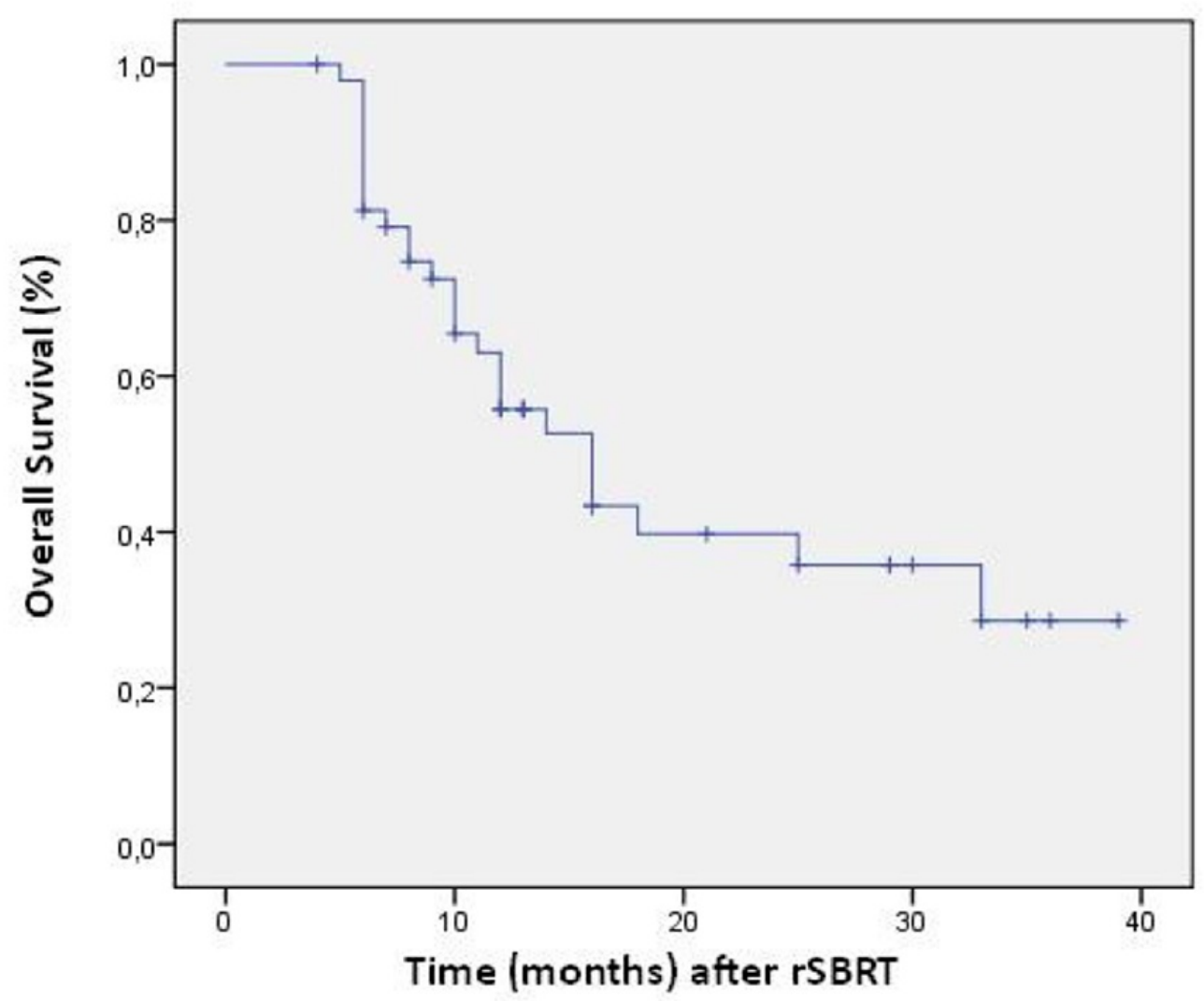

FIGURE 3: Actuarial overall survival rate for patients evaluated by the Kaplan-Meier method. 


\section{Cureus}

The actuarial one-year and two-year overall survival rates were 79\% (95\% (CI), 73-85\%) and $56 \%$ (95\% CI, 48-63\%), respectively. The overall survival rates were evaluated according to primary tumor histology, and statistically no difference was found between colorectal liver metastases and other primary tumor metastases ( $\mathrm{p}=0,085 \mathrm{Log}$ rank). The actuarial one-year overall survival rates were 70\% (95\% (CI), 59-81\%) and 67\% (95\% (CI), 56\%-77\%) and two-year were 52\% (95\% (CI), 38\%-66\%) and 37\% (95\% (CI), 25-50\%) were for colorectal liver metastases and other primary tumor metastases, respectively (Figure 4 ).

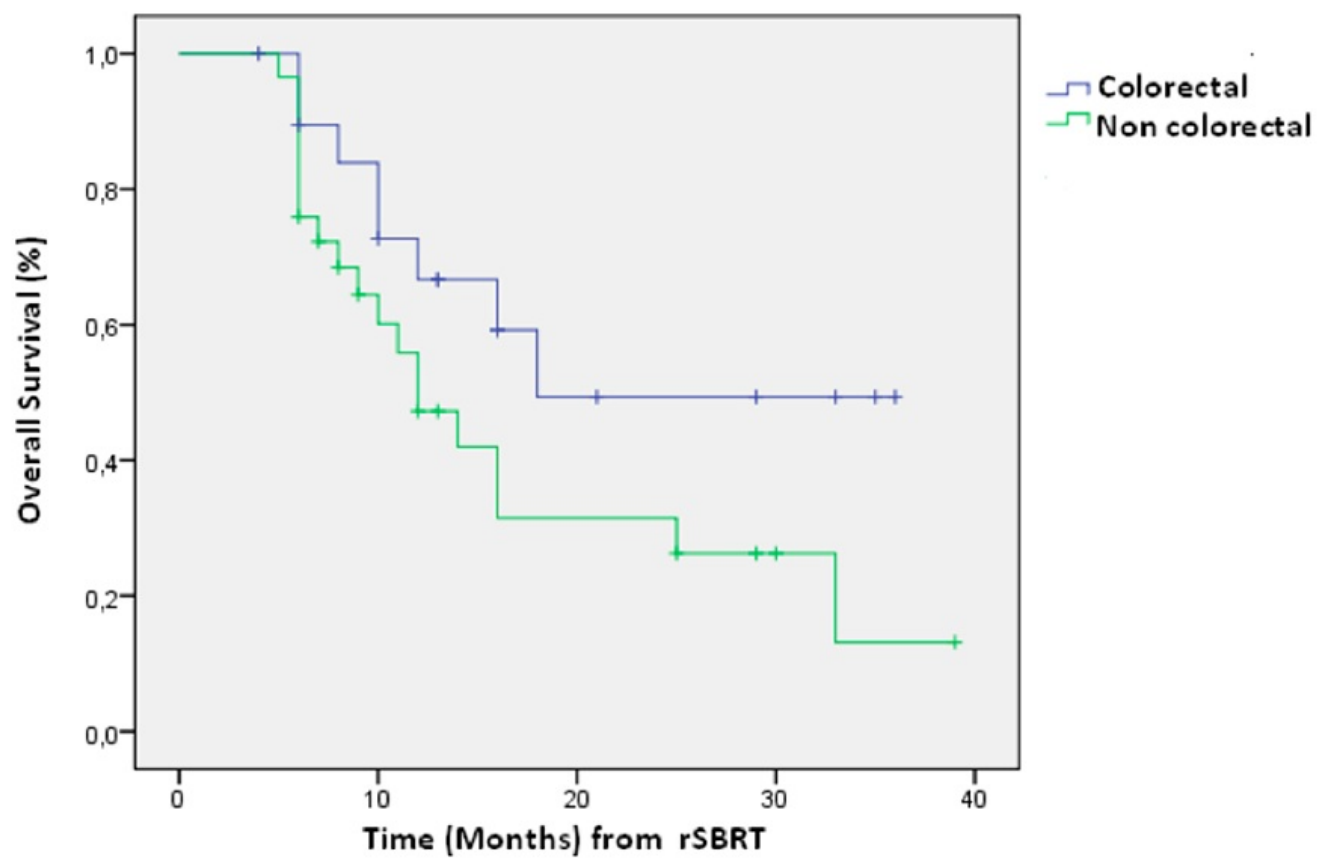

FIGURE 4: Actuarial overall survival rate by primary tumor types evaluated by the Kaplan-Meier method.

Kaplan Meier univariate analysis and Cox regression multivariate analysis were used for evaluating overall survival. Results are shown in Table 7 . Number of treated metastases was statically important for overall survival according to Cox regression multivariate analysis. Patients having one or two metastases treated had better survival than three metastases, according to our statistical results, as shown Figure 5.

Variable

Age

$\leq 60$

0.425

$>60$

Gender

\section{Univariate}

Multivariate

0.339

0.623

Male

0.827

0.839

1.115

Female 


\section{Cureus}

\# of treated lesion
1 or 2
0.127
0.011
6.085

3

Tumor size

$\leq 30 \mathrm{~mm}$

0.266

0.940

1.054

$>30 \mathrm{~mm}$

\# of fraction

$\leq 3$

0.293

0.072

3.817

$>3$

Total dose

$\leq 30$ Gy

0.485

0.547

1.428

$>30$ Gy

Primary histology

Colorectal

0.398

0.287

1.713

Noncolorectal

Other metastases
Yes
No

0.290

0.230

0.485

Concurrent chemoterapy

$$
\text { Yes }
$$

0.852

0.737

0.782

No

TABLE 7: Kaplan Meier univariate and Cox regression multivariate analysis for overall survival 


\section{Cureus}

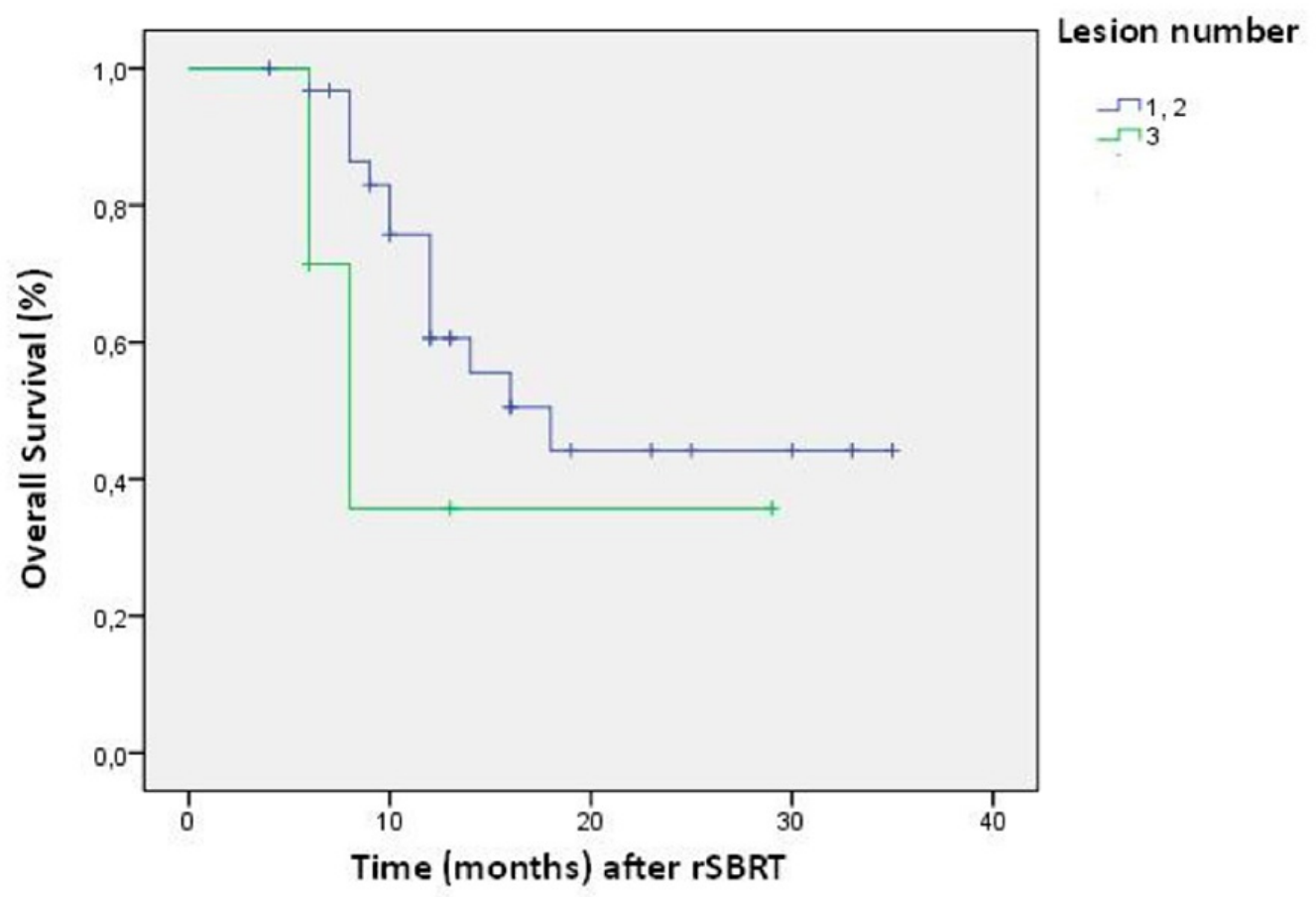

FIGURE 5: Actuarial overall survival rate number of treated lesion(s) evaluated by the Kaplan-Meier method.

\section{Toxicity}

Toxicity was evaluated in 49 patients according to NCI CTCAE, v3.0 criteria. All treatment was well-tolerated. No patient experienced dose-limiting toxicity. Acute and late toxicities of liver rSBRT are listed in Table 8. Hepatic pain and nausea were the most commonly observed toxicities. Fiducial migration was seen in five patients during rSBRT planning. Bleeding complication was observed in three patients during percutaneous implementation of fiducial markers. 


\section{Cureus}

\begin{tabular}{|c|c|c|c|c|c|c|}
\hline Follow up time & During rSBRT & & Acute & & Late & \\
\hline Toxicity grade & Grade 1,2 & Grade 3,4 & Grade 1,2 & Grade 3,4 & Grade 1,2 & Grade 3,4 \\
\hline Vomiting & 3 & 1 & 4 & & 2 & \\
\hline Nausea & 5 & 1 & 1 & & & \\
\hline Diarrhea & 3 & & 1 & & & \\
\hline Dermatitis & & & 3 & 1 & & \\
\hline Bleeding & 3 & & & & & \\
\hline Pain & 5 & & 4 & & 3 & \\
\hline Gastritis & & & 1 & & & \\
\hline Gastro duodenal & & & 1 & & & \\
\hline Fiducial migration & 5 & & & & & \\
\hline Evaluated \# of patients & 41 & & 32 & & 25 & \\
\hline
\end{tabular}

TABLE 8: Toxicity evaluation of rSBRT based on NCI CTCAE, v3.0 criteria.

Acute toxicity= Toxicity $\leq 3$ months, Late toxicity=Toxicity $>3$ months

\section{Discussion}

Liver is a common site of metastatic disease. The local control of oligometastatic disease might lead to improved systemic control. Several series have reported the outcomes after surgical resection of liver metastases [5-6, 22]. However, only a small portion of patients with liver metastases are candidates for surgical resection. In surgical series, resection of metastases increases median survival to approximately one year [4]. Patients with central lesions have the worst prognosis and the fewest therapeutic options. Other local ablative techniques or systemic therapies provide for a number of treatment options [10-14].

SBRT for liver metastases has been described in only a few studies [15-18, 23]. Different dose and fraction schema was tested in all these studies. These studies show that high local control rates can be achieved in patients with multiple liver metastases. In our study, the actuarial oneyear and two-year local control rates were 61\% (95\% confidence interval (CI), 53-69\%) and 39\% (95\% CI, 28-49\%), respectively. These results are similar to previously published series [4, 1618].

In this study involving rSBRT, the median overall survival was 16 months, a result which is similar to published series [16-18]. The actuarial one and two-year overall survival rates were $79 \%$ (95\% (CI), 73-85\%) and 56\% (95\% CI, 48-63\%), respectively. These results agree with the prior medical literature as reported by Wulf, et al. [24]; one and two-year overall survival rates were $76 \%$ and $61 \%$, respectively. Some factors, such as patient selection, additional metastases to other organs, etc., could adversely affect the local control and survival. Both Cox regression multivariate analysis for local control and survival show that number of treated lesion(s) is one 
the important parameters affecting treatment results. The effect of primary tumor histology and receiving systemic therapy were also observed in this study but these results are not significant. These parameters maybe need re-evaluation with larger patient populations in further studies.

\section{Conclusions}

The results of our study have shown that rSBRT for the treatment of liver metastases is feasible and safe with minimal side-effects. Further studies are required with larger patient series to compare rSBRT with other local ablative techniques and systemic therapies.

\section{Additional Information \\ Disclosures}

Human subjects: Consent was obtained by all participants in this study. Informed consent was obtained from all participants. The authors report no conflicts of interest. Animal subjects: All authors have confirmed that this study did not involve animal subjects or tissue. Conflicts of interest: In compliance with the ICMJE uniform disclosure form, all authors declare the following: Payment/services info: All authors have declared that no financial support was received from any organization for the submitted work. Financial relationships: All authors have declared that they have no financial relationships at present or within the previous three years with any organizations that might have an interest in the submitted work. Other relationships: All authors have declared that there are no other relationships or activities that could appear to have influenced the submitted work.

\section{References}

1. El-Serag HB, Rudolph KL: Hepatocellular carcinoma: Epidemiology and molecular carcinogenesis. Gastroenterology. 2007, 132:2557-2576.

2. Lau WY, Lai EC: Hepatocellular carcinoma: Current management and recent advances . Hepatobiliary Pancreat Dis Int. 2008, 7:237-257.

3. Wei AC, Greig PD., Grand D, et.al.: Survival after hepatic resection for colorectal metastases: a 10-year experience. Ann Surg Oncol. 2006, 13:668-676.

4. Alan WK, Madeline CS, Ann GM, et al.: Hypofractioned stereotactic body radiation therapy (SBRT) for limited hepatic metastases. Int J Radiation Oncology Biol Phys. 2007, 67:793-798.

5. Adam R, Vinet E: Regional treatment of metastasis.surgery of colorectal liver metastases . Annals of Oncology. 2004, 15:103-6.

6. Scheele J, Stang R, Altendorf-Hofmann A, et al.: Resection of colorectal liver metastases. World J Surg. 1995, 19:59-71.

7. Claire VD, Sylvain D, Francois B, et al.: Image-guided robotic stereotactic body radiation therapy for liver metastases: Is there a dose response relationship?. Int J Radiation Oncology Biol Phys. 2011, 81:e39-47.

8. Doci R, Gennari L, Bignami P, et al.: Morbidity and mortality after hepatic resection of metastases from colorectal cancer. Br J Surg. 1995, 82:377-381.

9. Fong Y, Fortner J, Sun RL, et al.: Clinical score for predicting,recurrence after hepatic resection for metastatic colorectal cancer: analysis of 1001 consecutive cases. Ann Surg. 1999, 230:309-318.

10. Bokemeyer C, Bondarenko I, Makhson A, et al.: Fluorouracil, leucovorin and oxaliplatin with and without cetuximab in the first-line treatment of metastatic colorectal cancer. J Clin Oncol. 2009, 27:663-671.

11. Meyerhardt JA, Mayer RJ: Drug therapy: Systemic therapy for colorectal cancer . N Engl J Med. 2005, 352:476-487.

12. Hurwitz H, Fehrenbacher L, Novotny W, et al.: Bevacizumab plus irinotecan, fluorouracil and leucovorin for metastatic colorectal cancer. New Engl J Med. 2004, 350:2335-2342.

13. de Baere T, Elias D, Dromain C, et al.: Radiofrequency ablation of 100 hepatic metastates with a mean follow-up of more than 1 year. Am J Roentgenol. 2000, 175:1619-1625. 
14. Shibata T, Niinobu T, Ogata N, et al.: Microwave coagulation therapy for multiple hepatic metastases from colorectal carcinoma. Cancer. 2000, 89:276-284.

15. Karyn AG, Ellen AW, Katherine EM, et al.: Dose-escalation study of single-fraction stereotactic body radiotherapy for liver malignancies. Int J Radiation Oncology Biol Phys. 2010, 78:486-493.

16. Giovanni A, Francesco P, Giorgio C, et al.: Image-guided robotic stereotactic radiosurgery for unresectable liver metastases: Preliminary results. Anticancer Research. 2009, 29:3381-3384.

17. Stintzing S, Hoffman RT, Heinemann V, et al.: Frameless single-session robotic radiosurgery of liver metastases in colorectal cancer patients. Eur J Cancer. 2010, 46:1026-1032.

18. Schefter TE, Kavanagh BD, Timmerman RD, et al.: A phase I trial of stereotactic body radiaition therapy (SBRT) for liver metastases. Int J Radiation Oncology Biol Phys. 2005, 62:1371-1378.

19. Pan CC, Kavanagh BD, Dawson LA, et al.: Radiation-associated liver injury. Int J Radiation Oncology Biol Phys. 2010, 76:S94-100.

20. Therasse P, Arbuck SG, Eisenhauer EA, et al.: New guidelines to evaluate the response to treatment in solid tumors. J Natl Cancer Inst. 2000, 92:205-216.

21. Common Terminology Criteria for Adverse Events (CTCAE) v3. . (2010). Accessed: 2010: http://www.eortc.be/services/doc/ctc/ctcaev3.pdf.

22. Bozzetti F, Cozzaglio L, Baracci P, et al.: Comparing surgical resection of limited hepatic metastases from colorectal cancer to non-operative treatment. Eur J Surg Oncol. 1993, 9:162167.

23. Wulf J, Guckenberger M, Haedinger U, et al.: Stereotactic radiotherapy of primary liver cancer and hepatic metastases. Acta Oncol. 2006, 45:838-847.

24. Wulf J, Hädinger U, Oppitz U, et al.: Stereotactic radiotherapy of targets in the lung and liver . Strahlenther Onkol. 2001, 177:645-655. 